\title{
Influence of the Mediterranean diet on the production of short-chain fatty acids in women at risk for breast cancer (LIBRE)
}

\author{
$\underline{\text { Benjamin Seethaler }}^{1}$, Jacqueline Beutel ${ }^{1}$, Marie Kogel ${ }^{1}$, Maryam Basrai ${ }^{1}$, Jens Walter ${ }^{2}$, \\ Martine Laville $^{3}$, Audrey M. Neyrinck ${ }^{4}$, Nathalie M. Delzenne ${ }^{4}$, Marion Kiechle ${ }^{5}$ and \\ Stephan C Bischoff ${ }^{1}$ \\ ${ }^{1}$ Institute of Nutritional Medicine, University of Hohenheim, Stuttgart, Germany, \\ ${ }^{2}$ Department of Agricultural, Food \& Nutritional Science and Department of Biological Sciences, University of Alberta, \\ Edmonton, Canada, \\ ${ }^{3}$ Rhône-Alpes Research Center for Human Nutrition, France, Lyon, France, \\ ${ }^{4}$ Metabolism and Nutrition Research Group, Louvain Drug Research Institute, Université catholique de Louvain, \\ Brussels, Belgium and \\ ${ }^{5}$ Department of Gynecology and Center for Hereditary Breast and Ovarian Cancer, Klinikum Rechts der Isar, \\ Technical University Munich (TUM) and Comprehensive Cancer Center Munich (CCCM), Munich, Germany
}

\begin{abstract}
Background: A number of small intervention studies suggested that a Mediterranean diet (MedD) and physical activity can lower the risk for breast cancer. LIBRE is the first large multicenter RCT to test the effect of these lifestyle factors on the incidence of breast cancer in women at risk because of BRCA mutations ${ }^{(1)}$. LIBRE also offers to unravel underlying mechanisms such as the role of short-chain fatty acids (SCFA) for beneficial effects of such lifestyle interventions.
\end{abstract}

Methods: We examined the effect of the lifestyle intervention on the production of SCFA measured in feces by gas chromatography. From the ongoing LIBRE trial we included all complete datasets (171 women; mean age $44 \pm 11$ years). Both women with and without previous breast cancer diagnosis were recruited (diseased; non-diseased). The participants were randomized into an intervention group (IG) trained for MedD and physical activity, and a usual care control group (CG). Adherence to the MedD was assessed at baseline and after 3 months (V1) using the validated Mediterranean Diet Adherence Screener (MEDAS) and the EPIC food frequency questionnaire (FFQ).

Results: At baseline there was no difference in SCFA levels between the groups. In the IG the MEDAS score increased substantially by 2.5 points $(p<0.001)$, in the $C G$ only mildly by 0.4 points $(p<0.05)$. Correspondingly, the intake of fibers increased solely in the IG. In the course of the study the amount of caproic acid decreased in the control group $(\mathrm{p}<0.001)$. At V1 non-diseased women showed higher amounts of acetic acid $(p=0.042)$, n-butyric acid $(p=0.023)$, n-valeric acid $(p=0.018)$ and iso-valeric acid $(p=$ 0.031). There were several correlations between the intake of different fibers and fecal SCFA. For example, the sum of poly- and oligosaccharides correlated with acetic acid $(p=0.001 ; r=0.316)$, propionic acid $(p=0.034 ; r=0,251), n-b u t y r i c$ acid $(p=0.010 ; r=$ $0.316)$ and iso-valeric acid $(p=0.012 ; r=0.306)$. There was no correlation between the MEDAS and SCFA.

Discussion: A lifestyle change towards a MedD and increased physical activity did not change the levels of SCFA in feces, although an increase of fiber intake was documented in the IG. To further analyze SCFA metabolism in this target population, gut microbiota composition and function (metabolites) are currently analyzed.

\section{Conflict of Interest}

There is no conflict of interest

\section{Reference}

1. Kiechle M, Engel C, Berling A, Hebestreit K, Bischoff SC, Dukatz R, Siniatchkin M, Pfeifer K et al.. (2016) Effects of lifestyle intervention in BRCA1/2 mutation carriers on nutrition, BMI, and physical fitness (LIBRE study): Study protocol for a randomized controlled trial. Trials 17, 368. 